\title{
An International Delphi Survey for the Definition of New Classification Criteria for Familial Mediterranean Fever, Mevalonate Kinase Deficiency, TNF Receptor-associated Periodic Fever Syndromes, and Cryopyrin-associated Periodic Syndrome
}

\author{
Silvia Federici (i), Federica Vanoni, Eldad Ben-Chetrit, Luca Cantarini, Joost Frenkel, \\ Raphaela Goldbach-Mansky, Ahmet Gul, Hal Hoffman, Isabelle Koné-Paut, \\ Jasmin Kuemmerle-Deschner, Helen J. Lachmann, Alberto Martini, Laura Obici, Seza Ozen, \\ Anna Simon, Michael Hofer, Nicolino Ruperto (D), and Marco Gattorno, for Eurofever \\ and the Pediatric Rheumatology International Trials Organization (PRINTO)
}

\begin{abstract}
Objective. Provisional evidence-based classification criteria for hereditary periodic fever (HPF) have been recently developed. However, no consensus on how to combine clinical criteria, laboratory tests, and results of molecular analysis has been reached. The objective of this study is to understand which variables physicians consider important for the classification of patients with HPF.

Methods. Two Delphi surveys were sent to health professionals in the field of autoinflammation. In the first open survey, 124 researchers could list all the variables they consider useful for the diagnosis of each monogenic periodic fever. The variables could be of any type and each researcher could complete the survey for 1 or more diseases. In the second survey, 162 researchers were asked to select, from a list of items coming from the first survey, the 10 top variables and to rank them by assigning a score from 10 to 1 .

Results. The response rates to the Delphi surveys were $85 \%$ for the first session and $87 \%$ for the second. The variables selected for each disease (corresponding to the third quartile, considering the total score obtained by the variables after the second Delphi survey) were 21 for mevalonate kinase deficiency, 22 for cryopyrinopathies, 18 for familial Mediterranean fever, and 20 for tumor necrosis factor receptorassociated periodic fever syndrome. A positive genetic test reached the top rank in all the HPF.

Conclusion. Our process led to the identification of those features considered the most important as candidate variables to be included in a new set of evidence-based classification criteria for HPF. (First Release November 1 2018; J Rheumatol 2019;46:429-36; doi:10.3899/jrheum.180056)
\end{abstract}

Key Indexing Terms:

CLASSIFICATION CRITERIA

MEVALONATE KINASE DEFICIENCY

FAMILIAL MEDITERRANEAN FEVER CRYOPYRINOPATHIES

TNF RECEPTOR-ASSOCIATED PERIODIC FEVER SYNDROMES

From the Istituto Giannina Gaslini, Clinica Pediatrica e Reumatologia, and Direzione Scientifica, PRINTO, Genoa, Italy; Ospedale Regionale di Bellinzona e Valli, Dipartimento di Pediatria, Bellinzona, Switzerland; Hadassah-Hebrew University Hospital, Rheumatology Unit, Jerusalem Israel; University of Siena, Department of Medical Sciences, Surgery and Neurosciences, Rheumatology Unit, Siena, Italy; Wilhelmina

Kinderziekenhuis, Universitair Medisch Centrum Utrecht, Afdeling Kinderreumatologie, Utrecht, the Netherlands; Rady Children's Specialists of San Diego, San Diego, California, USA, İstanbul Üniversitesi Cerrahpasa Tıp Fakültesi, Pediatrik Romatoloji, İstanbul, Turkey; Department of Pediatric Rheumatology, National Referral Centre of AutoInflammatory Diseases, CEREMAI, Centre Hospitalier Universitaire (CHU) de Biĉetre, AP-HP, University of Paris Sud, le Kremlin Bicetre, France; Division of Rheumatology, Department of Pediatrics and Autoinflammation Reference Center Tuebingen, University Hospital Tübingen, Tübingen, Germany; Royal Free Campus, National Amyloidosis Centre, London, UK; Fondazione Istituto di Ricovero e Cura a Carattere Scientifico (IRCCS) Policlinico San Matteo, Centro per lo Studio e la Cura delle Amiloidosi Sistemiche, Pavia, Italy; Hacettepe University,
Department of Pediatric Rheumatology, Ankara, Turkey; Radboud University Medical Center (UMC), Pediatric Rheumatology, Nijmegen, the Netherlands; Unité Romande d'Immuno-rhumatologie Pédiatrique, Centre hospitalier universitaire vaudois (CHUV), University of Lausanne, Lausanne; Geneva University Hospitals, Geneva, Switzerland.
This study is part of the International Study Group for Systemic Autoinflammatory Diseases project, funded by the E-Rare-3 program, grant number 9003037603.
S. Federici, MD, PhD, Istituto Giannina Gaslini, Clinica Pediatrica e Reumatologia, PRINTO; F. Vanoni, MD, Ospedale Regionale di Bellinzona e Valli, Dipartimento di Pediatria, and Geneva University Hospitals; E. Ben-Chetrit, MD, Hadassah-Hebrew University Hospital, Rheumatology Unit; L. Cantarini, MD, PhD, University of Siena, Department of Medical Sciences, Surgery and Neurosciences, Rheumatology Unit; J. Frenkel, MD, PhD, Wilhelmina Kinderziekenhuis, Universitair Medisch Centrum Utrecht, Afdeling Kinderreumatologie; R. Goldbach-Mansky, MD, MHS, Rady Children's Specialists of San Diego; A. Gul, MD, İstanbul Üniversitesi Cerrahpaşa Tıp Fakültesi, 
Pediatrik Romatoloji; H. Hoffman, MD, Rady Children's Specialists of San Diego; I. Koné-Paut, MD, Department of Pediatric Rheumatology,

National Referral Centre of Auto-Inflammatory Diseases CEREMAI, CHU de Bicetre, AP-HP, University of Paris Sud, le Kremlin Bicetre;

J. Kuemmerle-Deschner, MD, Division of Rheumatology, Department of Pediatrics and Autoinflammation Reference Center Tübingen, University Hospital Tübingen; H.J. Lachmann, MD, Royal Free Campus, National Amyloidosis Centre; A. Martini, MD, Istituto Giannina Gaslini, Direzione Scientifica; L. Obici, MD, Fondazione IRCCS Policlinico San Matteo, Centro per lo Studio e la Cura delle Amiloidosi Sistemiche; S. Ozen, MD, Hacettepe University, Department of Pediatric Rheumatology; A. Simon, MD, PhD, Radboud UMC, Pediatric Rheumatology; M. Hofer, MD, Unité Romande d'Immuno-rhumatologie Pédiatrique, CHUV, University of Lausanne, and Geneva University Hospitals; N. Ruperto, MD, MPH, Istituto Giannina Gaslini, Clinica Pediatrica e Reumatologia, PRINTO; M. Gattorno, MD, Istituto Giannina Gaslini, Clinica Pediatrica e Reumatologia, PRINTO. These authors contributed equally to this article: S. Federici, F. Vanoni, N. Ruperto, and M. Gattorno.

Address correspondence to Dr. S. Federici, Istituto Giannina Gaslini, Pediatric Division, Via Gaslini 5, Genoa 16147, Italy.

E-mail: silviafederici@gmail.com

Accepted for publication July 18, 2018.

The hereditary periodic fever (HPF) syndromes are a group of monogenic disorders manifesting with recurrent episodes of fever lasting from few to several days and accompanied by systemic inflammation and organ-specific manifestations. Several diseases are included under this term, such as the familial Mediterranean fever (FMF), mevalonate kinase deficiency (MKD), the tumor necrosis factor (TNF) receptorassociated periodic fever syndrome (TRAPS), and cryopyrin-associated periodic syndrome (CAPS). These diseases in turn include a range of conditions from the mildest familial cold autoinflammatory syndrome (FCAS) through the intermediate Muckle-Wells syndrome to the chronic infantile neurological cutaneous and articular syndrome (CINCA) or neonatal-onset multisystem inflammatory disease, which represent the most severe forms of the spectrum. FMF is the most common monogenic autoinflammatory syndrome because of the gain of function mutation on the $M E F V$ gene. FMF attacks usually last from 12 to $72 \mathrm{~h}$ and are characterized by serosal inflammation causing chest and abdominal pain, arthralgia/arthritis, and erysipeloid erythematous rash. TRAPS is inherited as an autosomal dominant disease due to mutations in the TNFRSF1A gene. The episodes are longer lasting, from 1 to 4 weeks or more, and are characterized by variable skin manifestations (maculopapular, urticarial, or erythematous migratory rash), myalgia, fasciitis, abdominal pain, periorbital edema, or conjunctivitis. Amyloidosis, although less common than in untreated FMF, may lead to renal failure. MKD is caused by mutations in the $M V K$ gene, which encodes mevalonate kinase. Attacks last 3 to 7 days and are often precipitated by triggering factors such as immunizations, surgery, trauma, or mild infections. Gastrointestinal (GI) manifestations with abdominal pain, nausea/vomiting, and diarrhea often dominate the clinical picture, along with possible maculopapular rash, cervical lymphadenopathy, or splenomegaly. CAPS includes a wide spectrum (from the milder FCAS to the most severe CINCA) of clinical manifestations caused by dominantly inherited missense mutations in the NLRP3 gene. A number of diagnostic and classification clinical criteria for HPF are available in the literature and used in clinical practice, but all of them present some limitations, mainly related to the methodology used, the patient's sample characteristics, or the absence of the results of genetic analysis. Additionally, there is not a clear distinction between diagnostic criteria, which usually rely on pathognomic findings (e.g., genetic analysis, glycemia for patients with diabetes, etc.), and classification criteria, which are aimed to identify a set of clinical/laboratory/genetic findings with high sensitivity and specificity ${ }^{1,2}$.

The overall aim of this project was to obtain a large consensus on the development of novel, more sensitive and specific classification criteria for FMF, MKD, TRAPS, and CAPS through consensus-formation technique and data validation in the large dataset of the Eurofever Registry ${ }^{3,4}$. In this manuscript, we report the results of the first step of the process aimed to identify, by an international Delphi consensus, the candidate measures for the proper classification of each of these conditions.

\section{MATERIALS AND METHODS}

For the development of the HPF classification criteria, we used a multistep approach. The first step (Delphi) relates to the identification of the most important variables that could be used to classify each of the diseases. In the second step (classification and analysis), we asked a selected panel of clinicians/researchers to classify individual patients from Eurofever as per each HPF (reference standard) and then analyze the Eurofever database to derive a series of classification criteria for each HPF. In the third and final step, we selected the final classification criteria in a consensus conference. This article reports the first of these steps.

Step 1: Delphi questionnaire surveys. The Delphi technique uses a series of well-defined mail questionnaires, with the first open to avoid any biases, and the subsequent ones based on the results of the prior ones.

The surveys have been conducted through a secure Web-based system with the technical help of the Pediatric Rheumatology International Trials Organization (PRINTO at www.printo.it) ${ }^{5}$. Participants were pediatric/adult rheumatologists involved in their daily clinical practice of treating patients with autoinflammatory diseases.

The first Delphi questionnaire was sent by e-mail to centers belonging to the PRINTO network actively enrolling patients in the Eurofever registry ${ }^{3}$.

Independent ethical approval for entering patients in the Eurofever registry and consent for participation was obtained in the participating countries, in accordance with local requirements. Approval at the Coordinating Center (Istituto G. Gaslini) was obtained (protocol number $1-17 / 03 / 2015)$. Consent was obtained, asking permission to use clinical data for research purposes.

Clinicians and researchers working in the field of autoinflammatory diseases were asked to indicate by free text the variables thought most likely to be helpful and relevant in their clinical and research practice for the diagnosis/classification of FMF, TRAPS, MKD, and CAPS. The questionnaire was anonymous, and in the letter of invitation all the experts were asked to give their permission to analyze and publish data coming from the survey. Variables to be included could be of any type and each responder could fill the survey for 1 or more diseases on the basis of his/her expertise. Two reminders were sent every 6 weeks to all investigators who had not replied.

From the first survey, a list of clinical and laboratory variables were obtained with redundancies deleted. All remaining variables were grouped

Personal non-commercial use only. The Journal of Rheumatology Copyright @ ${ }^{2019}$. All rights reserved. 
into mutually exclusive categories, and within each category, listed in alphabetical order (no rank provided). Additional missing variables derived from the already published classification/diagnostic criteria for any disease were eventually included in the list derived from the first Delphi open survey.

The second Delphi questionnaire was sent to all those invited to participate in the previous survey except to those who declined further involvement. In this phase, 43 American clinicians dealing with autoinflammatory diseases, not participating to the Eurofever registry but members of Paediatric Rheumatology Collaborative Study Group (PRCSG) and the Childhood Arthritis and Rheumatology Research Alliance (CARRA), were also involved. Participants were first asked to choose, among the variables listed in the first Delphi survey, the top 10 they consider the most important for the classification of each periodic fever syndrome. In a second step, they were asked to rank the previously selected items from 1 to 10 , where 1 was the least important and 10 the most important. Each rank could be used only once even though some features were thought to be equally important. At the end of the questionnaire, the participants could add any missing feature from the list that they considered as relevant.

After the second Delphi survey, a list of variables, including any chosen at least once by the participants, was obtained for each disease. The sum of ranks, frequency of ranking, and median score with first and third quartile was calculated for each variable. Those variables falling in the higher third quartile, considering the total score obtained, were selected and will be used in the second steps of the study as previously described.

\section{RESULTS}

The first survey was sent to 124 participants coming from 63 countries. A total of 106/124 (85\%) responded to the initial invitation and 84/106 (79\%) completed and confirmed it (Figure 1).
A total of 73/84 clinicians completed the survey for FMF, 55 for TRAPS, 65 for CAPS, and 55 for MKD.

The number of variables obtained for each disease at the end of the first Delphi survey was 104 for FMF, 98 for TRAPS, 108 for CAPS, and 116 for MKD (Supplementary Table 1, available with the online version of this article).

The candidate variables cited for all the diseases, checked for redundancies, were classified in 5 mutually exclusive categories: history, characteristic of fever episodes, signs and symptoms, laboratory test, and other investigations (Supplementary Table 1, available with the online version of this article). All the variables present in the published criteria were cited by at least 1 participant in the survey.

The second survey was sent to 162 people, 118 from the PRINTO network and 44 from CARRA/PRCSG. The overall rate of response was 141/162 researchers, and among these, 120 completed the survey. In particular, 109 participants completed the survey for FMF, 93 for TRAPS, 102 for CAPS, and 94 for MKD (Figure 2).

At the end of the second Delphi survey, the variables chosen at least once by any participants were 275 (Supplementary Table 2, available with the online version of this article), 70/104 for FMF, 76/98 for TRAPS, 81/108 for CAPS, and 81/116 for MKD. The remaining variables have not been ranked by any of the participants. Moreover, none of the participants added any variables to the list proposed,

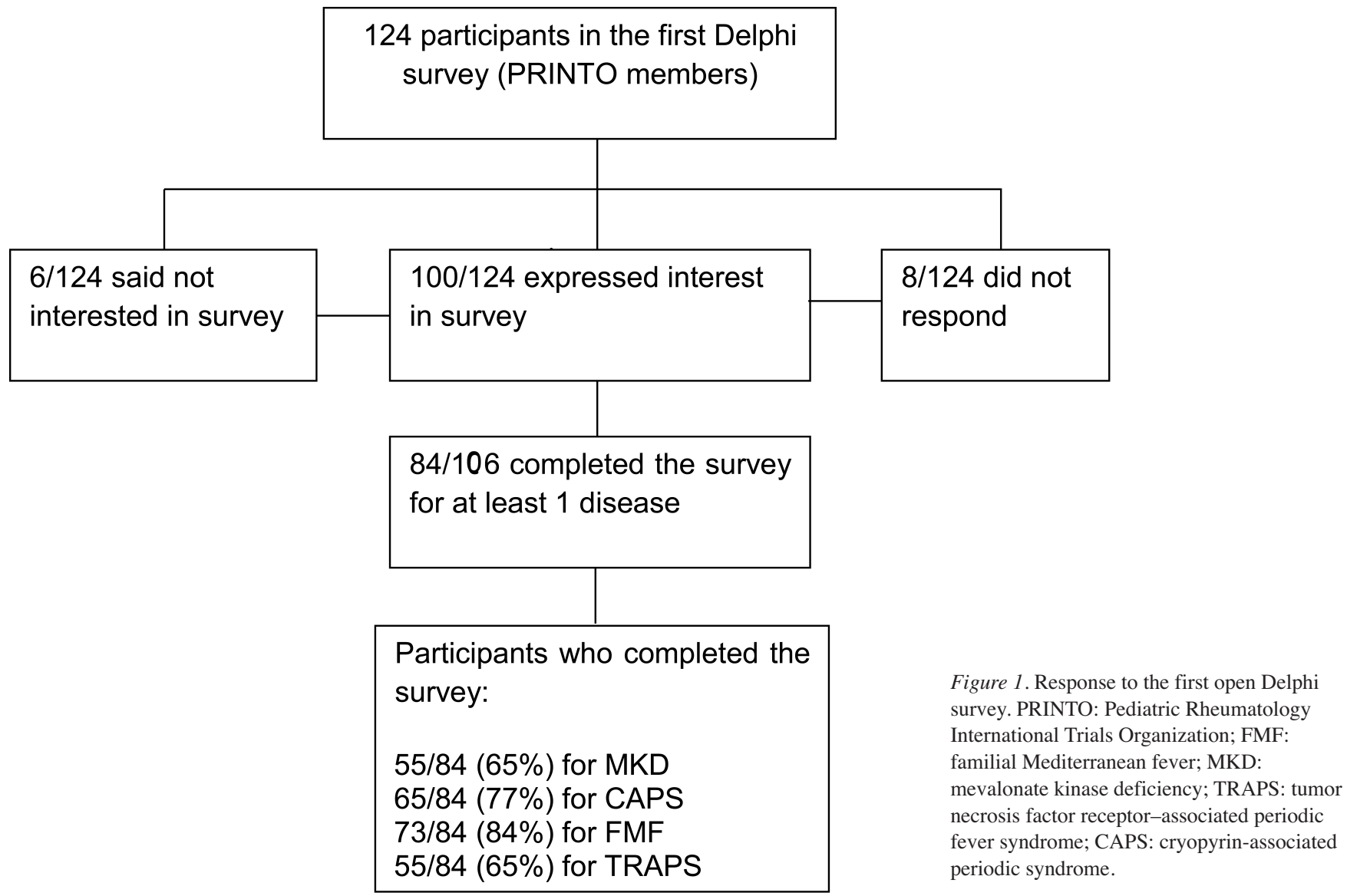




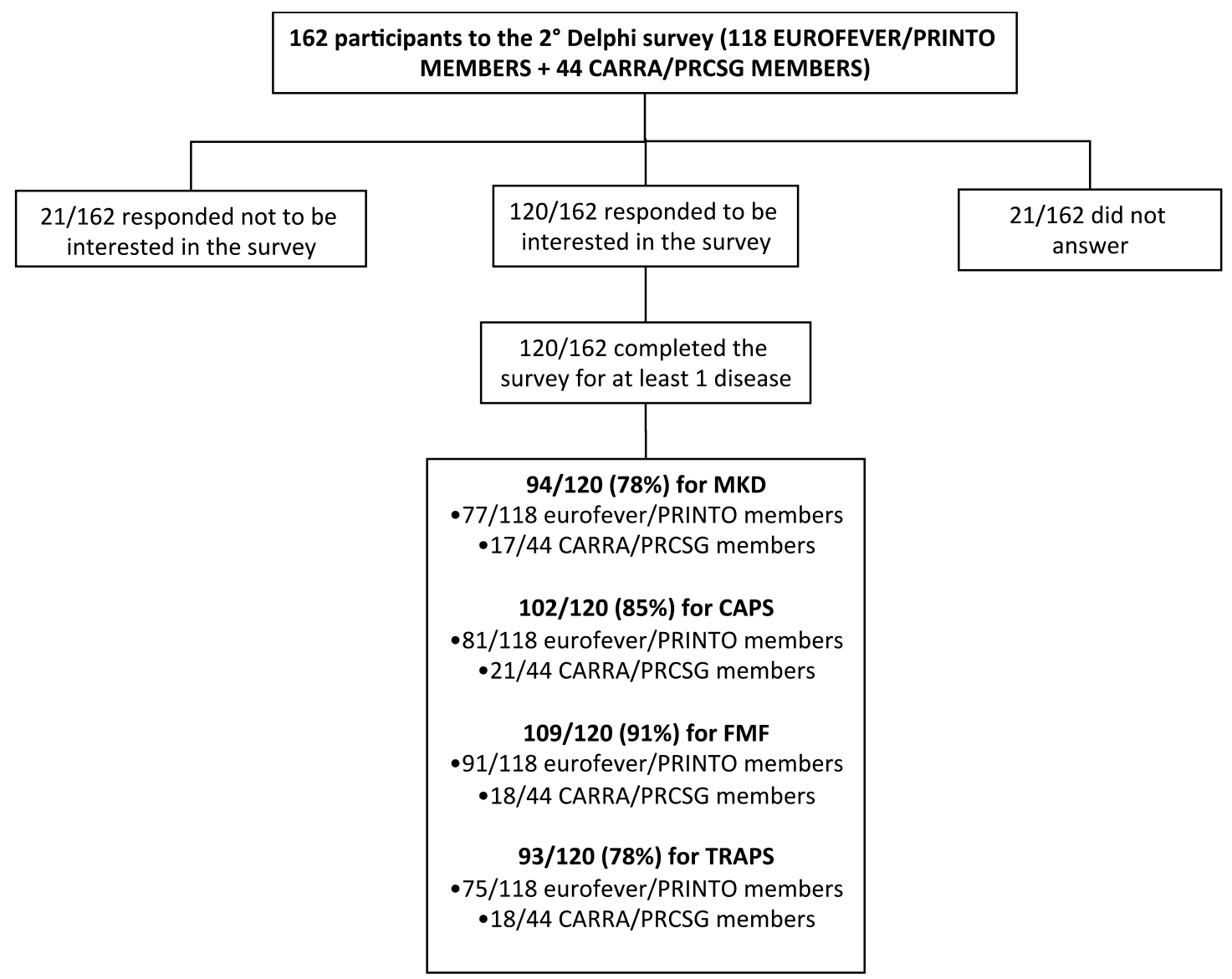

Figure 2. Response to the second open Delphi survey. PRINTO: Pediatric Rheumatology International Trials Organization; FMF: familial Mediterranean fever; MKD: mevalonate kinase deficiency; TRAPS: tumor necrosis factor receptor-associated periodic fever syndrome; CAPS: cryopyrin-associated periodic syndrome; CARRA: Childhood Arthritis and Rheumatology Research Alliance; PRCSG: Paediatric Rheumatology Collaborative Study Group.

suggesting that participants agreed that the items coming from the first survey were exhaustive.

For the subsequent statistical analysis, we selected those variables falling in the third quartile considering the total score obtained, thus including 18 variables for FMF (Table 1), 20 for TRAPS (Table 2), 21 for MKD (Table 3), and 22 for CAPS (Table 4). The ranking, including frequencies of selection and medium score for each disease, is shown in Tables 1-4.

For all HPF, the presence of a "positive genetic test" for the causative gene was the variable with the highest rank.

Among the clinical manifestations, the most cited variables for FMF have been abdominal pain, southeast Mediterranean ethnicity, duration of fever episodes between 1 to 3 days, serositis, and erysipeloid rash (Table 1). Classified in the highest rank for TRAPS were the presence of recurrent long-lasting fever episodes associated with positive family history, periorbital edema, abdominal pain, myalgia, cutaneous rash, monocytic fasciitis, and conjunctivitis (Table 2). For MKD, the early age of onset, the presence of triggering factors, cervical lymphadenopathy (often painful), and GI manifestation (abdominal pain, diarrhea, vomiting) received the highest rank (Table 3). For CAPS, urticarial rash reached the second position, followed by hearing loss, early age of onset, and chronic meningitis (Table 4).

The increase of acute-phase reactants during fever episodes (and in some cases their persistence between episodes) also received a high rank in all the diseases (Tables 1-4).

The determination of mevalonic acid in the urine and the reduction of enzymatic activity reached the second and 17 th positions (Table 3) for MKD, respectively, whereas the response to colchicine and interleukin 1 (IL-1) blockers reached the second and third position, respectively, for FMF and CAPS. For TRAPS and MKD, none of the variables indicating a response to a specific therapy reached a total score sufficient to be ranked in the third quartile. In these diseases, the response to steroids reached, respectively, the 22nd and 34th positions, while a response to anti-TNF agent reached the 24th rank for TRAPS.

A number of variables falling in the third quartile indicated by the Delphi survey were not included in the diagnostic/

Personal non-commercial use only. The Journal of Rheumatology Copyright $@$ (0) 2019. All rights reserved. 
Table 1. Variables falling in the third quartile, considering the total score obtained for familial Mediterranean fever (FMF).

\begin{tabular}{|c|c|c|c|c|}
\hline Rank & Variable & Score & $\begin{array}{l}\text { Frequency } \\
\text { of Citation }\end{array}$ & $\begin{array}{l}\text { Mean } \\
\text { Score }\end{array}$ \\
\hline 1 & Positive genetic analysis for $M E F V$ gene & 626 & 84 & 7.5 \\
\hline 2 & Response to colchicine ${ }^{\wedge} *$ & 568 & 87 & 6.5 \\
\hline 3 & $\begin{array}{l}\text { Increase of acute-phase reactants and serum amyloid A } \\
\text { during fever episodes* }{ }^{\circ}\end{array}$ & 471 & 77 & 6.1 \\
\hline 4 & Abdominal pain* $* \S$ & 466 & 84 & 5.5 \\
\hline 5 & Ethnicity (Turkish, Arabs, Armenian, Kurds, Jewish) $* \S$ & 426 & 66 & 6.5 \\
\hline 6 & Classic recurrent fever pattern^ ${ }^{\wedge} \circ$ & 378 & 46 & 8.2 \\
\hline 7 & Duration of attacks $1-3$ days*。 & 313 & 42 & 7.5 \\
\hline 8 & Positive family history^ $* \circ$ & 253 & 51 & 5.0 \\
\hline 9 & Serositis $^{\wedge} *$ & 220 & 36 & 6.1 \\
\hline 10 & Erysipeloid rash^${ }^{\wedge}$ & 198 & 47 & 4.2 \\
\hline 11 & Self-limiting episodes* & 181 & 35 & 5.2 \\
\hline 12 & Duration of attacks few hours to $3-4$ days* ${ }^{\circ}$ & 166 & 22 & 7.5 \\
\hline 13 & Chest pain*०§ & 152 & 35 & 4.3 \\
\hline 14 & Arthritis*。 & 127 & 32 & 4.0 \\
\hline 15 & Well-being between episodes* & 124 & 32 & 3.9 \\
\hline 16 & Amyloidosis^ & 104 & 20 & 5.2 \\
\hline 17 & $\begin{array}{l}\text { Increase of acute-phase reactants and serum amyloid A } \\
\text { during fever episodes and sometimes between episodes }\end{array}$ & 100 & 17 & 5.9 \\
\hline 18 & Arthralgia & 80 & 24 & 3.3 \\
\hline
\end{tabular}

* Variable already present in Livneh criteria. ${ }^{\wedge}$ Tel Hashomer criteria. ${ }^{\circ}$ Pediatric FMF criteria. ${ }^{\S}$ Preliminary Eurofever criteria.

Table 2. Variables falling in the third quartile, considering the total score obtained for tumor necrosis factor receptor-associated autoinflammatory syndrome (TRAPS).

\begin{tabular}{|c|c|c|c|c|}
\hline Rank & Variable & Score & $\begin{array}{l}\text { Frequency } \\
\text { of Citation }\end{array}$ & $\begin{array}{l}\text { Mean } \\
\text { Score }\end{array}$ \\
\hline 1 & Positive genetic analysis for TNFRSF $1 A$ gene & 637 & 74 & 8.6 \\
\hline 2 & $\begin{array}{l}\text { Increase of acute-phase reactants and serum amyloid A } \\
\text { during fever episodes }\end{array}$ & 323 & 50 & 6.5 \\
\hline 3 & Recurrent prolonged episodes of fever & 301 & 35 & 8.6 \\
\hline 4 & Periorbital edema & 286 & 55 & 5.2 \\
\hline 5 & Positive family history ${ }^{\S}$ & 282 & 48 & 5.9 \\
\hline 6 & Irregular, long-lasting fever episodes & 254 & 31 & 8.2 \\
\hline 7 & Abdominal pain & 251 & 57 & 4.4 \\
\hline 8 & Myalgia ${ }^{\S}$ & 176 & 39 & 4.5 \\
\hline 9 & Fever lasting more than 7 days ${ }^{\S}$ & 169 & 23 & 7.3 \\
\hline 10 & Localized intense myalgia ${ }^{\S}$ & 135 & 23 & 5.9 \\
\hline 11 & $\operatorname{Rash}^{\S}$ & 129 & 32 & 4.0 \\
\hline 12 & Migratory rash ${ }^{\S}$ & 128 & 27 & 4.7 \\
\hline 13 & Duration of attacks $1-3$ weeks & 121 & 15 & 8.1 \\
\hline 14 & Arthralgia & 119 & 32 & 3.7 \\
\hline 15 & Monocytic fasciitis & 114 & 17 & 6.7 \\
\hline 16 & $\begin{array}{l}\text { Increase of acute-phase reactants and serum amyloid A } \\
\text { during fever episodes and between episodes }\end{array}$ & 114 & 17 & 6.7 \\
\hline 17 & Fever lasting more than 5 days $^{\S}$ & 86 & 10 & 8.6 \\
\hline 18 & Conjunctivitis & 86 & 20 & 4.3 \\
\hline 19 & Recurrent episodes of fever & 82 & 10 & 8.2 \\
\hline 20 & Painful maculopapular rash & 82 & 12 & 6.8 \\
\hline
\end{tabular}

$\S$ Variable already present in preliminary Eurofever criteria. 
Table 3. Variables falling in the third quartile, considering the total score obtained for mevalonate kinase deficiency (MKD).

\begin{tabular}{|c|c|c|c|c|}
\hline Rank & Variable & Score & $\begin{array}{l}\text { Frequency } \\
\text { of Citation }\end{array}$ & $\begin{array}{l}\text { Medium } \\
\text { Score }\end{array}$ \\
\hline 1 & Positive genetic test for $M V K$ gene & 637 & 73 & 8.7 \\
\hline 2 & Increased urinary mevalonic acid during episodes & 352 & 54 & 6.5 \\
\hline 3 & Duration of attacks $3-7$ days & 328 & 49 & 6.7 \\
\hline 4 & Fever $^{\S}$ & 327 & 42 & 7.8 \\
\hline 5 & Disease onset $<1 \mathrm{yr}^{\S}$ & 317 & 39 & 8.1 \\
\hline 6 & $\begin{array}{l}\text { Increase of acute-phase reactants and serum amyloid A } \\
\text { during fever episodes }\end{array}$ & 276 & 48 & 5.8 \\
\hline 7 & Increased IgD levels & 252 & 45 & 5.6 \\
\hline 8 & $\begin{array}{l}\text { Presence of triggering factors (immunization, infection, } \\
\text { minor trauma, surgery) }\end{array}$ & 218 & 35 & 6.2 \\
\hline 9 & Abdominal pain & 184 & 45 & 4.1 \\
\hline 10 & Lymphadenopathy (often painful) ${ }^{\S}$ & 150 & 31 & 4.8 \\
\hline 11 & Early disease onset ${ }^{\S}$ & 139 & 25 & 5.6 \\
\hline 12 & Cervical lymphadenopathy & 137 & 31 & 4.4 \\
\hline 13 & Gastrointestinal manifestation ${ }^{\S}$ & 113 & 21 & 5.4 \\
\hline 14 & Diarrhea $^{\S}$ & 106 & 26 & 4.1 \\
\hline 15 & Maculopapular rash & 85 & 19 & 4.5 \\
\hline 16 & Rash & 83 & 21 & 4.0 \\
\hline 17 & Mevalonate kinase activity & 80 & 12 & 6.7 \\
\hline 18 & Aphthosis $\S$ & 80 & 21 & 3.8 \\
\hline 19 & Disease onset $<2$ yrs $^{\S}$ & 73 & 12 & 6.1 \\
\hline 20 & Irregular periodicity & 73 & 19 & 3.8 \\
\hline 21 & Self-limiting episodes & 72 & 15 & 4.8 \\
\hline
\end{tabular}

$\S$ Variable already present in the preliminary Eurofever criteria.

Table 4. Variables falling in the third quartile, considering the total score obtained for cryopyrin-associated periodic syndrome (CAPS).

\begin{tabular}{|c|c|c|c|c|}
\hline Rank & Variable & Score & $\begin{array}{l}\text { Frequency } \\
\text { of Citation }\end{array}$ & $\begin{array}{l}\text { Medium } \\
\text { Score }\end{array}$ \\
\hline 1 & Positive genetic analysis for $N L R P 3$ gene & 554 & 71 & 7.8 \\
\hline 2 & Urticarial rash ${ }^{\S}$ & 479 & 66 & 7.3 \\
\hline 3 & Response to IL-1 $\beta$ blockade & 469 & 69 & 6.8 \\
\hline 4 & Recurrent fever ${ }^{\S}$ & 434 & 55 & 7.9 \\
\hline 5 & $\begin{array}{l}\text { Increase of acute-phase reactants and serum amyloid A } \\
\text { during fever episodes \# }\end{array}$ & 331 & 52 & 6.4 \\
\hline 6 & Hearing loss $\S^{\S \#}$ & 314 & 57 & 5.5 \\
\hline 7 & Episodes triggered by cold exposure ${ }^{\#}$ & 291 & 50 & 5.8 \\
\hline 8 & Age at onset $<1 \mathrm{yr}$ & 185 & 28 & 6.6 \\
\hline 9 & Chronic urticaria ${ }^{\S}$ & 177 & 29 & 6.1 \\
\hline 10 & Chronic meningitis ${ }^{\#}$ & 171 & 39 & 4.4 \\
\hline 11 & $\begin{array}{l}\text { Increase of acute-phase reactants and serum amyloid A } \\
\text { during fever episodes and between episodes }\end{array}$ & 166 & 28 & 5.9 \\
\hline 12 & Chronic disease course & 165 & 29 & 5.7 \\
\hline 13 & Fever & 157 & 22 & 7.1 \\
\hline 14 & Positive family history & 151 & 34 & 4.4 \\
\hline 15 & Eye involvement ${ }^{\S}$ & 104 & 19 & 5.5 \\
\hline 16 & Neurologic involvement ${ }^{\#}$ & 102 & 18 & 5.7 \\
\hline 17 & Positive NLRP12 genetic test & 94 & 14 & 6.7 \\
\hline 18 & Conjunctivitis ${ }^{\S}$ & 85 & 20 & 4.3 \\
\hline 19 & Osteoarthropathy" & 74 & 14 & 5.3 \\
\hline 20 & Arthralgia & 70 & 24 & 2.9 \\
\hline 21 & Cartilage overgrowth" & 66 & 14 & 4.7 \\
\hline 22 & Age at onset $<1 \mathrm{mo}$ & 66 & 11 & 6.0 \\
\hline
\end{tabular}

$\S$ Variable already present in the Eurofever criteria. ${ }^{\#}$ Variable already present in the CAPS criteria. IL: interleukin. 
classification criteria available so far (Tables 1-4). As already shown, a positive genetic test reached the first rank in all diseases.

The elevation of acute-phase reactants was considered as a criterion in the recently proposed CAPS criteria only (Table 4). Moreover, MVK enzymatic activity, urinary secretion of mevalonic acid, and high IgD serum levels were considered important for the classification of MKD (Table 3).

Finally, the Delphi was able to identify a number of clinical manifestations not included in previous criteria, especially for TRAPS (abdominal pain, arthralgia, monocytic fasciitis; Table 2) and MKD (early onset, presence of triggering factors, abdominal pain, rash; Table 3). A positive response to anti-IL-1 treatment was included as possible diagnostic/classification criteria for CAPS (Table 4).

We then analyzed the possible differences in the rank given to each variable falling in the top quartile between European and American clinicians. Both groups indicated the same variables in the higher positions, although in a slightly different order. Notably, in all diseases, the positive genetic test reached the top position in both groups (Supplementary Table 3, and Supplementary Table 4, available with the online version of this table).

\section{DISCUSSION}

To our knowledge, this is the first Delphi survey for the identification of candidate variables for a new set of classification criteria for monogenic periodic fever syndromes. The good rate of response to the Delphi underlines the interest of clinicians in this field.

To date, a number of diagnostic/classification criteria for some HPF are already available in the literature. Nonetheless, most of them have been created based on the judgment of a limited group of experts, mainly in countries with a dominant prevalence of a single disease, such as FMF in southeastern Mediterranean countries.

For example, the 2 historical criteria for FMF, Tel-Hashomer ${ }^{6}$ and Livneh criteria ${ }^{7}$, were created in the Israeli population before the identification of the gene responsible for the disease. Similarly, the more recent pediatric FMF criteria ${ }^{8}$ were developed in the Turkish population. The main limitation of the current FMF criteria is related to their low accuracy when tested in populations in which the other monogenic diseases display a similar prevalence ${ }^{9}$. This is mainly due to the evident overlap among the different conditions that share a number of clinical manifestations.

To overcome these limitations, preliminary evidence-based criteria for monogenic HPF were developed from the Eurofever registry ${ }^{10}$. These criteria have been built on the basis of a statistical analysis conducted on a large cohort of real adult and pediatric patients affected by different HPF who were enrolled in the registry. These criteria were expressed as a score with a cutoff based on the best performance in a validation set of patients. The high accuracy of these criteria was mainly related to the presence of either "positive" and "negative" criteria for each condition. This means that in these latter criteria are included symptoms whose presence is indicative for the disease and symptoms whose absence increases the risk of being affected ${ }^{10}$.

Recently, Kuemmerle-Deschner and co-workers developed a new set of clinical diagnostic criteria for CAPS that were validated in the context of a number of confounding diseases ${ }^{11}$.

In our present study we took advantage of the Delphi technique to involve a large number of clinicians dealing with the management of patients with systemic autoinflammatory diseases worldwide.

We obtained a list of variables considered useful for the classification of these patients, ranked in order of importance. Interestingly, in all diseases the higher rank was obtained by the presence of a positive genetic test, even for those diseases, such as FMF, in which the diagnosis is frequently based on clinical ground, especially in the Mediterranean countries displaying a high incidence of this condition.

A number of laboratory examinations was also considered. Elevation of acute-phase reactants resulted in the first 6 positions in all HPF. So far, the only diagnostic criteria including laboratory examinations are those recently proposed for CAPS, in which the elevation of acute-phase reactants in association to the clinical manifestations is considered a mandatory criterion ${ }^{11}$.

Despite the limitation of their availability in all centers, both the urinary secretion of mevalonic acid during fever episodes and determination of impaired MVK enzymatic activity were considered to play a relevant role in the diagnosis/classification of MKD. The same was suggested for the high serum IgD levels, even if previous studies already showed their low sensitivity and specificity ${ }^{12}$.

Overall, the results obtained by the Delphi survey reflect the current clinical practice for the diagnosis of HPF. Although in some countries the diagnosis of periodic fever is still based only on clinical features, the current overall approach is certainly that of a combination of clinical, genetic, and laboratory data.

The strong indication coming from clinicians involved in the field is therefore to avoid criteria based exclusively on clinical variables, emphasizing the importance of including genetic and laboratory variables in new classification criteria.

This indication seems particularly true in the next-generation sequencing era, in which the increasing availability of genetic testing will increase the need to integrate data coming from the genetic analysis with the clinical manifestations. Of course, not all variants associated to a given gene can be consider as pathognomonic. In fact, hypomorphic variants (polymorphisms, low-penetrance mutations), variants of uncertain pathogenic significance, and incomplete genotypes (i.e., heterozygous status in autosomal recessive diseases) represent future possibilities.

Personal non-commercial use only. The Journal of Rheumatology Copyright $\odot$ 2019. All rights reserved 
To avoid possible misinterpretation of data coming from molecular testing, we will need (1) a precise characterization of the actual pathogenicity of each variant associated to HPF genes, and (2) novel evidence-based criteria based on the combination of clinical manifestations and data coming from molecular analysis. These 2 aims will represent the actual goal of the future steps for the identification of novel evidence-based classification criteria.

This article reports the results from an international Delphi survey involving a large number of experts dealing with HPF from several countries. The process allowed the identification of those features that the experts considered the most important candidate variables to be included in a new set of evidence-based classification criteria for HPF. The next steps of this project will include the analysis of the performance of the variables chosen by the present Delphi in real patients, in accordance with the judgment of experienced clinicians and geneticists. A final consensus on the best combination of clinical, laboratory, and genetic variables will allow for the final identification of the best evidence-based classification criteria to be used in the clinical setting.

\section{ACKNOWLEDGMENT}

The authors acknowledge Eugenia Mosci, Elisa Patrone, and Roberto Cavanna for technical support.

\section{ONLINE SUPPLEMENT}

Supplementary material accompanies the online version of this article.

\section{REFERENCES}

1. Hunder GG, Arend WP, Bloch DA, Calabrese LH, Fauci AS, Fries JF, et al. The American College of Rheumatology 1990 criteria for the classification of vasculitis. Introduction. Arthritis Rheum 1990;33:1065-7.
2. Aggarwal R, Ringold S, Khanna D, Neogi T, Johnson SR, Miller A, et al. Distinctions between diagnostic and classification criteria? Arthritis Care Res 2015;67:891-7.

3. Toplak N, Frenkel J, Ozen S, Lachmann HJ, Woo P, Koné-Paut I, et al. An international registry in autoinflammatory diseases: the Eurofever experience. Ann Rheum Dis 2012;71:1177-82.

4. Ozen S, Frenkel J, Ruperto N, Gattorno M; Eurofever Project. The Eurofever Project: toward better care for autoinflammatory diseases. Eur J Pediatr 2011;170:445-52.

5. Ruperto N, Martini A, for the Paediatric Rheumatology International Trials Organisation (PRINTO). Networking in pediatrics: the example of the Pediatric Rheumatology International Trials Organisation (PRINTO). Arch Dis Child 2011;96:596-601.

6. Sohar E. Tel Hashomer criteria for the diagnosis of FMF. First International Conference on FMF. London and Tel Aviv: Freund Publishing House; 1997:207.

7. Livneh A, Langevitz P, Zemer D, Zaks N, Kees S, Lidar T, et al. Criteria for the diagnosis of familial Mediterranean fever. Arthritis Rheum 1997;40:1879-85.

8. Yalçinkaya F, Ozen S, Ozçakar ZB, Aktay N, Cakar N, Düzova A, et al. A new set of criteria for the diagnosis of familial Mediterranean fever in childhood. Rheumatology 2009;48:395-8.

9. Demirkaya E, Saglam C, Turker T, Koné-Paut I, Woo P, Doglio M, et al. Performance of different diagnostic criteria for familial Mediterranean fever in children with periodic fevers: results from a multicenter international registry. J Rheumatol 2016;43:154-60.

10. Federici S, Sormani MP, Ozen S, Lachmann HJ, Amaryan G, Woo P, et al. Evidence-based provisional clinical classification criteria for autoinflammatory periodic fevers. Ann Rheum Dis 2015; 74:799-805.

11. Kuemmerle-Deschner JB, Ozen S, Tyrrell PN, Kone-Paut I, Goldbach-Mansky R, Lachmann H, et al. Diagnostic criteria for cryopyrin-associated periodic syndrome (CAPS). Ann Rheum Dis 2017;76:942-7.

12. Ammouri W, Cuisset L, Rouaghe S, Rolland MO, Delpech M, Grateau G, et al. Diagnostic value of serum immunoglobulinaemia $D$ level in patients with a clinical suspicion of hyper IgD syndrome. Rheumatology 2007;46:1597-600 